\title{
Let Digons be Bygones: The Fate of Excitons in Curved $\pi$-Systems
}

Dianelys Ondarse, ${ }^{\dagger}$ Tammie Nelson, ${ }^{\ddagger}$ John M. Lupton, ${ }^{\top}$ Sergei Tretiak, ${ }^{\ddagger}$ and Sebastian Fernandez Alberti*, $\dagger$

Universidad Nacional de Quilmes/CONICET, Roque Saenz Peña 352, B1876BXD Bernal, Argentina, Theoretical Division, Physics and Chemistry of Materials (T-1), Los Alamos

National Laboratory, Los Alamos, New Mexico 87545, USA, and Institut für Angewandte und Experimentelle Physik, Universität Regensburg, Universitätsstrasse 31, 93053

Regensburg (Germany)

E-mail: sfalberti@gmail.com

${ }^{*}$ To whom correspondence should be addressed

${ }^{\dagger}$ Universidad Nacional de Quilmes

‡Los Alamos National Laboratory

ฯUniversität Regensburg 


\begin{abstract}
We explore the diverse origins of unpolarized absorption and emission of molecular polygons, consisting of $\pi$-conjugated oligomer chains held in a bent geometry by strain controlled at the vertex units. For this purpose, we make use of atomistic nonadiabatic excited-state molecular dynamics simulations of a bichromophore molecular polygon (digon) with bent chromophore chains. Both structural and photoexcited dynamics were found to affect polarization features. Bending strain induces exciton localization on individual chromophore units of the conjugated chains. The latter display different transition dipole moment orientations, a feature not present in the linear oligomer counterparts. In addition, bending makes exciton localization very sensitive to molecular distortions induced by thermal fluctuations. The excited-state dynamics reveals an ultrafast intramolecular energy redistribution that spreads the exciton equally among spatially separated chromophore fragments within the molecular system. As a result, digons become virtually unpolarized absorbers and emitters, in agreement with recent experimental studies on the single-molecule level.
\end{abstract}

\title{
Graphical TOC Entry
}

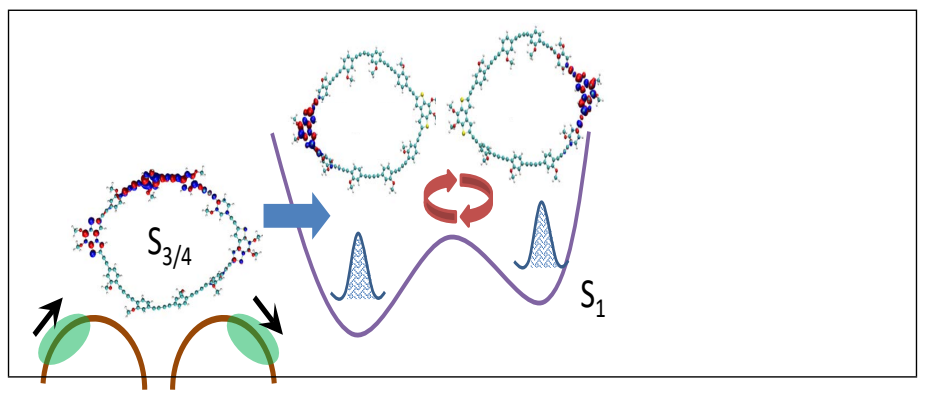


Molecular geometry plays an important role in determining macroscopic properties that affect the performance and efficiency of optoelectronic devices. Geometry is particularly crucial in soft organic materials, such as $\pi$-conjugated polymers and chromophores, commonly used in applications such as organic light-emitting diodes (OLEDs) and organic photovoltaics (OPV). ${ }^{1-3}$ The underlying $\pi$-conjugated backbone in these molecules makes them highly susceptible to thermal fluctuations that can alter their shape through bending and/or twisting (torsion) motions. ${ }^{4,5}$ Furthermore, unlike the exciton splitting commonly observed in strong and intermediate coupling regimes, weakly coupled systems are typically characterized by quasi-degenerate excitations originating from the individual chromophores. These factors result in the spatial localization of excitations. As a consequence, energy levels in weakly coupled systems tend to be very sensitive to changes in morphology and geometry fluctuations. ${ }^{6-13}$ This sensitivity, in turn, perturbs intra- and intermolecular interactions. Polymer thin films have highly variable morphologies. Along with interchain interactions, photophysical properties of these amorphous materials are highly dependent on chain packing, which directly affects device performance. ${ }^{14}$

Such effects ultimately derive from changes in the geometry of a single chain that induce changes in interchain interactions and collective morphology. This impact has been confirmed in phenylene-vinylenes, where the actual shape of the individual chromophore dominates the spectroscopic properties and not their collective arrangement. ${ }^{15}$ The changes in individual chromophore geometry imply a concomitant change in the molecular dipole moment. In particular, the molecular dipole moment is significant in determining electronic properties of interfaces ${ }^{16-19}$ and has been exploited to improve device efficiencies through inclusion of strong-dipole spacer layers in donor-acceptor solar cells. ${ }^{20}$

Similarly, the orientation of the transition dipole moment (TDM) is also sensitive to geometry distortions and morphology. ${ }^{21,22}$ The TDM defines absorption and fluorescence properties of the material. For example, the TDM orientation is well known to be involved in controlling the efficiency of emission in OLEDs. ${ }^{23,24}$ Furthermore, the alignment 
of TDMs between two weakly coupled molecules - head-to-tail (J-aggregates) or side-to-side (H-aggregates) - has been demonstrated to determine the spectral properties and radiative decay rates. ${ }^{25,26}$ For example, misaligned TDMs between monomer units of coupled conjugated polymer chains arises from chain bending and allows the photoluminescence peak ratios to be selectively tuned. Thus chain bending can provide control over the radiative characteristics of conjugated polymer chains which can be useful in OLEDs design. ${ }^{26}$

Dynamic processes, such as electronic energy transfer among chromophores, can induce reorientation of the transition dipole. Time resolved fluorescence anisotropy decay is used to quantify the evolution of the transition dipole orientation. It is commonly applied to study excitation localization and migration in photosynthetic ${ }^{27,28}$ and artificial light harvesting systems including nanorings, ${ }^{29}$ macrocycles, ${ }^{30,31}$ dendrimers, ${ }^{32}$ conjugated polymers, ${ }^{4,33,34}$ and chromophore dimers, ${ }^{35,36}$ to name a few, and can be directly simulated by nonadiabatic excited state molecular dynamics. ${ }^{37-40}$ The question of how bending may affect the TDM orientation of the species being probed, and whether it can make absorption isotropic, remains open. The fluorescence anisotropy decay is caused by changes in the orientation of the chromophores relative to their initial orientation upon photoexcitation. The ultrafast depolarization response is a strong argument for the process of coherent intrachain energy transfer. ${ }^{33}$ The same response could also be generated if the chromophores themselves are bent. For simplicity, however, chromophores are typically assumed to be straight. ${ }^{33}$ Neglecting the geometry of the chain in the interpretation of experimental data could potentially cause the response associated with chain bending to be erroneously attributed to other processes such as interchromophoric energy transfer.

Molecular polygons provide a very useful testbed system for investigating the effect of chromophore bending on energy transfer and anisotropy. They consist of $\pi$-conjugated oligomers held in a bent geometry where the strain is controlled by the opening angle of the vertex unit and the oligomer chain length. Details of the synthesis and optical characteristics of several molecular polygons have previously been reported. ${ }^{41}$ Their spectroscopic charac- 
terization revealed unique photophysical behavior as a result of the bent chromophores. Bending of the $\pi$-system, which is clearly visualized in real space scanning-tunneling microscopy images of single molecules, was found to induce structural relaxation in the excited state, increasing the Stokes' shift and vibronic coupling in photoluminescence. In a linear system, the overall TDM is comprised of individual segments adding up, whereas bending potentially causes cancellation of the contributions from the individual monomers. Exciton localization within the chain due to torsional motions of the segments will also affect the TDM orientation in bent chromophores, but not in their linear counterparts. The TDM cancellation is expected to increase the radiative relaxation lifetime, while exciton localization should have the opposite effect by lowering the total oscillator strength and decreasing radiative relaxation lifetimes in bent systems. The overall process of excited state relaxation is difficult to predict in bent $\pi$-conjugated systems due to a complex interplay between these competing effects.

Nonadiabatic excited state molecular dynamics (NEXMD) simulations $^{42-45}$ were performed to model the photoexcited dynamics and non-radiative relaxation of the bichromophore molecular polygon (digon) with bent chromophores whose chemical structure is shown in Figure 1a. The digon is composed of two phenylene-ethynylene-butadiynylene chains linked at both ends by bithiophene vertices. The simulated equilibrated absorption spectrum shown in Figure 1b is given by the histograms of the vertical transition energies calculated from the ensemble of initial ground state geometries, where the height is defined by oscillator strengths and transition frequencies. ${ }^{46}$

While the simulated spectrum is slightly red shifted compared to experiment (See Figure $\mathrm{S} 1$ ), the key features are captured by simulation. The experimental absorption peak, centered around $375 \mathrm{~nm}$, is broadened at lower energy consistent with the $S_{1} / S_{2}$ state vertex absorption that corresponds to the shoulder in the simulated spectrum. We choose our excitation wavelength to be consistent with experiment by exciting the simulated absorption peak at $432 \mathrm{~nm}$ corresponding to chain-localized states. The contributions from individual 
excited states shown in Figure 1b overlap strongly. Therefore, considering a realistic laser pulse width, it is impossible to excite a single state but rather a range of states are initially populated in the ensemble. The simulated absorption peak at $432 \mathrm{~nm}$ can be primarily attributed to the range of states $S_{3}-S_{5}$, whose specific spatial localization depends on the molecular conformation at the moment of laser excitation. In order to determine the electronic character of the exciton, we analyzed the initial localization of states $S_{1}-S_{5}$ from the transition density distributions. Since the specific spatial localization depends on the molecular conformation, transition density distributions among the different ground state equilibrated structures are analyzed rather than the ground state minimum. The transition density distributions shown in Figure S2 confirm that the lowest energy states $S_{1}$ and $S_{2}$ are localized in opposite vertex units and the higher energy states $S_{3}-S_{5}$ become increasingly localized on the chains. The broad distributions shown in Figure S2 indicate that exciton localization among vertices and chains of the digon is very sensitive to molecular conformations. This sensitivity is more pronounced in $S_{3}-S_{5}$ high-energy states. Since these states give rise to the initial excitation, the resulting exciton localization will be strongly dependent on the conformational diversity of the molecule at room temperature. This feature contributes to the digon's behavior as an unpolarized molecular absorber. ${ }^{41}$

While $S_{3}-S_{5}$ states are indeed localized on the chains, the exciton can initially form on different segments due to torsional distortions along the oligomer chain that break conjugation. Therefore, we do not distinguish the initial states by their adiabatic labels, instead we consider the variation of initial localization within the chain. Overall, the initial excitation of bent chains results from contributions of individual chromophoric units within the chain whose TDMs experience mutual cancellations to different degrees. This effect is illustrated in Figure 2 where the spatial distribution of initial electronic transition densities is depicted for six examples of initial geometries from our NEXMD simulations. Owing to the conformational variability due to thermal fluctuations and strain, the localization arises at different parts of the chains for each excitation event. A further analysis of the exciton 

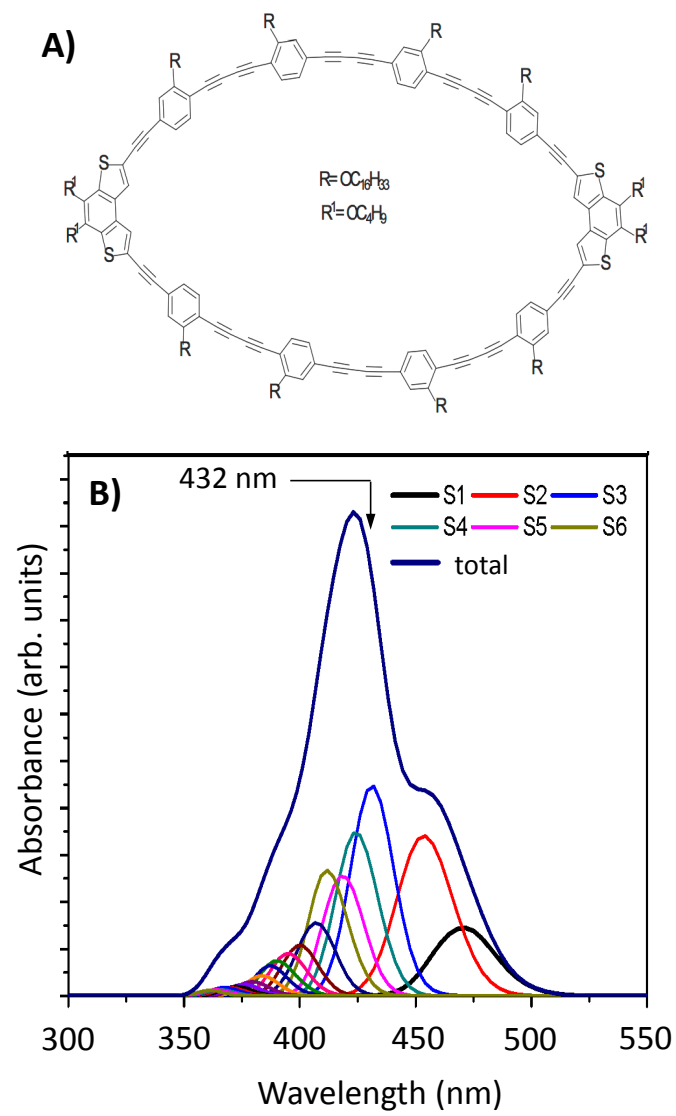

Figure 1: a) Chemical structure of the phenylene-ethynylene-butadiynylene-based digon with bithiophene end units (vertices) having an opening angle of $101^{\circ}$. b) The equilibrated absorption spectrum of the digon at $300 \mathrm{~K}$ showing contributions from 20 individual excited states $\left(S_{1}-S_{6}\right.$ labeled) to the total spectrum.

localization throughout different segments of the chains can be seen in Figure S3 showing the distribution within the chain of whichever state initially absorbs the most. There we see that the localization is distributed along the chains in an asymmetric way between the inner/outer and left/right chromophores of the chain. We conclude that bending reduces the effective conjugation in the excited states and, therefore, it promotes exciton localization on different fragments of the conjugated chain, leading to an effectively unpolarized absorption of light by the digons.

After an initial laser excitation at $432 \mathrm{~nm}$ with populates mainly $S_{3}$ and $S_{4}$ states, the digon experiences ultrafast sequential internal conversion $S_{4 / 3} \longrightarrow S_{2} \longrightarrow S_{1}$ (see Figure 3(a)). Since the $S_{4 / 3}, S_{2}$, and $S_{1}$ states are characterized by different spatial localization, 

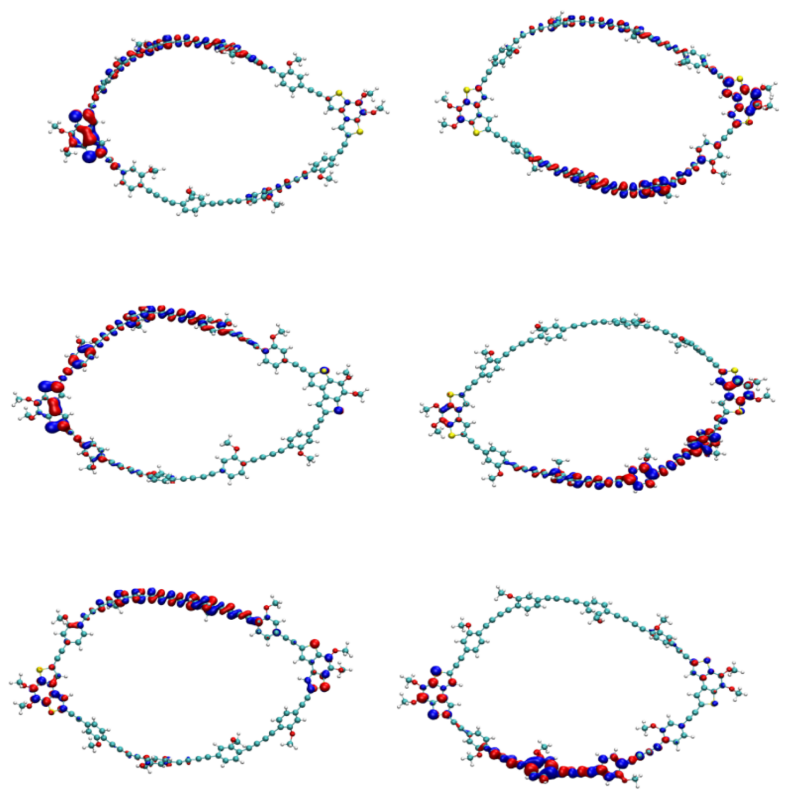

Figure 2: Transition density plots of the initial exciton localization for different initial geometries. The representative snapshots were obtained from 1 ns equilibrated ground-state molecular dynamics simulations at 300K.

the molecular system also undergoes ultrafast intramolecular spatial redistribution of excitation energy. This redistribution of the exciton wave function is evident in the evolution of the average transition density localization in different parts of the molecule shown in Figure 3(b). To analyze the localization of transition density, we classified chains and vertices as follows: We define "high" and "low" chains as chains with the highest and lowest fraction of electronic transition density $(\delta)$, respectively, at the initial time ( $\mathrm{t}=0 \mathrm{fs}$ ). Aditionally, we denote "high" and "low" vertices as vertices with the highest and lowest fraction of electronic transition density $(\delta)$, respectively, at the final time ( $\mathrm{t}=500 \mathrm{fs})$. The redistribution occurs from the high chain where the exciton is initially localized to the low chain and finally to the vertices (see Figure 3(b)). As shown in Figure 3 (inset), both vertices initially acquire equal amounts of excitation energy. These exciton redistribution processes introduce another source of polarization anisotropy in addition to the depolarization originating from the initial unpolarized absorption discussed above. As a consequence, after the initial 150 fs of excited-state dynamics, the exciton no longer remains on the conjugated chains (except for 

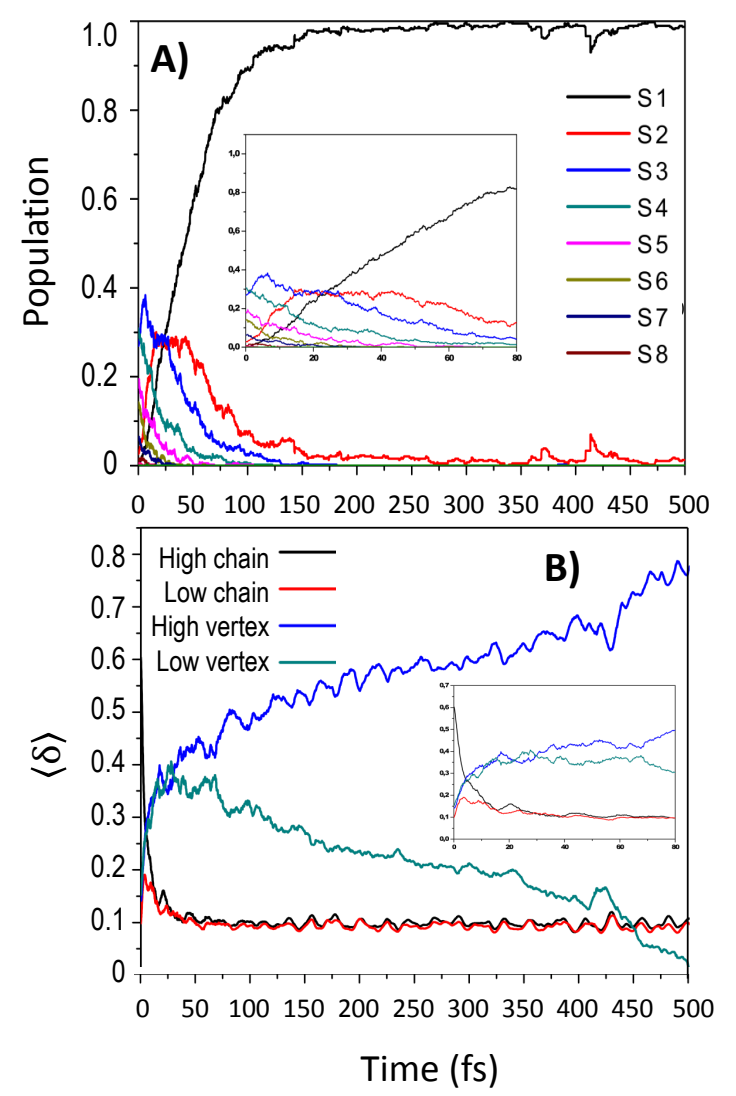

Figure 3: a) Evolution of adiabatic state populations during NEXMD dynamics for states $S_{1}-S_{8}$ participating in the relaxation. Populations are calculated as the fraction of trajectories with a given state occupation at each time. b) Evolution of the fraction of transition density averaged over the ensemble of trajectories for the "high" chain with the highest initial transition density (black) and the "low" chain with the lowest transition density (red) as well as the "high" vertex with the highest final transition density (blue) and the "low" vertex with the lowest final transition density (green).

about $10 \%$ population), but gets transferred onto the vertices. Thereafter, a slower throughspace energy transfer takes place between vertices until the exciton finally becomes localized (self-trapped) on a single (high) vertex. At the final time of our simulations (500 fs), the localization of the exciton on the vertices presents an asymmetric distribution of $\sim 80 \%$ on one vertex and less than $\sim 5 \%$ on the other. It is important to stress that this through-space exciton exchange between vertices persists even though the molecule has entirely relaxed to the lowest $S_{1}$ state, as can be seen by comparing Figure 3(a) and (b). 


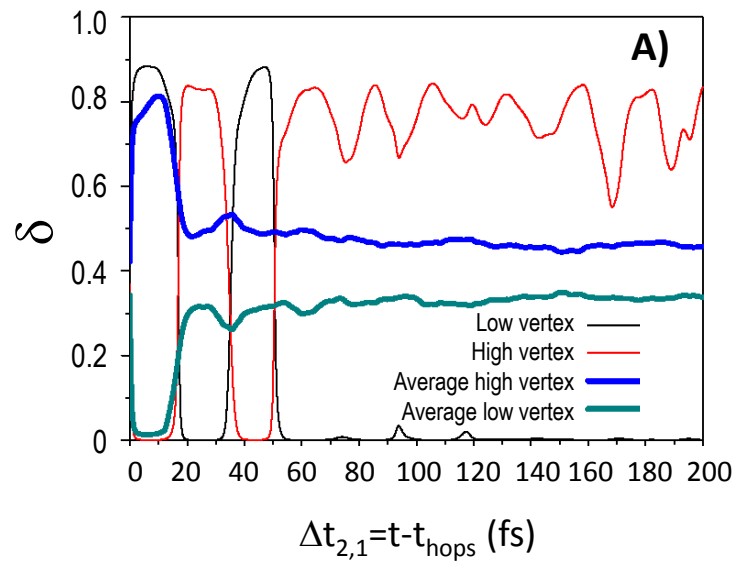

B)
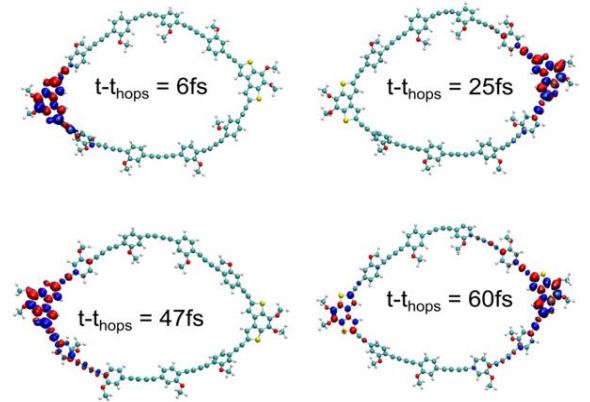

C)

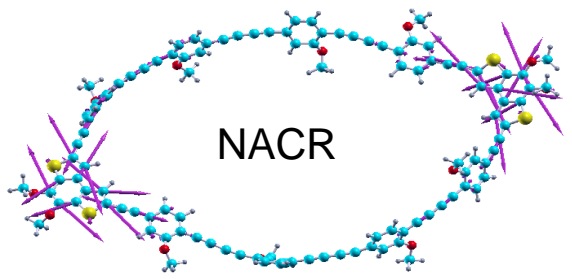

Figure 4: a) Evolution of the fraction of transition density in the two vertices after the nonadiabatic transition to $S_{1}$ for an individual trajectory (red and black lines) revealing oscillations of the electronic localization between the vertices. The average over the ensemble of trajectories shows that oscillatory behavior dephases within 100fs. b) Snapshots of the transition density along the individual trajectory reveal the coherent oscillation of the wave function between the vertex units following the final effective hop to $S_{1}$. c) Nonadiabatic coupling vector (NACR) between $S_{1}$ and $S_{2}$ responsible for the nonadiabatic transition, which involves mutual asymmetric displacements of atoms on the vertex units.

Figure 4 shows that nonadiabatic transitions $S_{2} \longrightarrow S_{1}$ induce oscillations of the electronic wave function between vertices. In order to synchronize the nonadiabatic transitions among individual trajectories, we introduce a time variable $\Delta t=t-t_{h o p s}$. The exact time of transition varies among trajectories, and is set to $\Delta t=0$ in this time frame. Negative values of $\Delta t$ correspond to times before the nonadiabatic transition and positive values represent times after the transition. The time axis in Figure 4(a) denotes the time from the 
final effective hop $S_{2} \longrightarrow S_{1}$. After the transition $\left(t-t_{\text {hops }}>0\right)$, the system on the lower state experiences oscillations between the two vertices before becoming trapped on a single vertex. That is, the vertices interchange the exciton through space in a coherent way, as is particularly evident in the evolution of the single trajectory. On average, there are 2-3 hops between the vertices (see Figure S4). These oscillations are the result of the asymmetric nuclear motions that the direction of the nonadiabatic coupling vector (NACR) experiences on opposite vertices (see Figure 4(c)). This coherent exciton-vibrational coupling behavior has been reported previously to arise during the energy transfer process within several different molecular complexes, which presented equivalent asymmetric forms of NACRs. ${ }^{47}$ The bent conjugated chains here act as rigid bridges between the two equivalent chromophores (vertices), without actively participating in the specific vibrational motions involved in the $S_{2} \longrightarrow S_{1}$ relaxation process.

In summary, photoinduced dynamics in molecular polygons composed of bent $\pi$-conjugated oligomer chains has been analyzed using nonadiabatic excited-state molecular dynamics simulations. Digons offer a controllable well-defined molecular system allowing the study of the effect of bending by fixing the oligomers in a strained geometry. This approach introduces an interplay of isotropy and anisotropy effects on the photoexcited dynamics. Our results reveal a variety of features responsible for the virtually unpolarized absorption and emission observed experimentally. ${ }^{41}$ First, we confirm that bent conjugated chains localize excitons on individual chromophore units ensuring different transition dipole moment orientations, a feature not present in the linear counterparts. Furthermore, the initial exciton localization is very sensitive to molecular distortions induced by thermal fluctuations. Both of these features make digons unpolarized light absorbers. Evidence of virtually unpolarized emission has been seen experimentally in the linear dichroism histograms of digons. ${ }^{41}$ We have found the additional effect that comes from an ultrafast intramolecular energy redistribution that equally spreads the exciton, initially localized on different fragments of the bent conjugated chains, to the different chromophores spatially localized on different parts of the molecule, 
i.e., the opposite bent conjugated chain and vertices.

These exciton redistribution processes are ultrafast and precede the timescale of the emission. The excited state localizes rapidly $(\sim 50 \mathrm{fs})$ on one of the two vertex units of the digon, presumably due to the strong conjugation of the thiophene unit. From there, coherent vibronic dynamics induces out-of-phase oscillations of the exciton wave function between the vertices immediately following the $S_{2} \longrightarrow S_{1}$ nonadiabatic transition. Subsequently, a coherent exchange between the two vertex units emerges, mediated by the asymmetry of the NACR, and emission ultimately arises from the vertex unit where $S_{1}$ is localized in accordance with the Kasha's rule. ${ }^{48}$ These coherent dynamics result in effective excited state isotropy that can explain the observation of virtually unpolarized emission as follows. The net orientation of the transition dipole moment will be extremely sensitive to the degree of localization, so that every excitation event will give rise to a slightly different transition dipole moment orientation. In addition, the localization to either of the vertex units is non-deterministic, so that even if the fluctuations of the dipole orientation on one vertex (between subsequent localization events) are not very large, a substantial difference will exist between localization on either end of the digon in terms of the net dipole orientation. The end result will be a tendency for virtually unpolarized emission. Rather than achieving nearly unpolarized emission through localization fluctuations on the conjugated oligomers, it is a result of the highly conjugated thiophene vertex units acting as a sort of trapping site. This raises interesting avenues to explore the effect of altering the vertex unit composition which can change both the trapping ability of the vertices as well as the bending strain on the oligomer chains. 


\section{Computational Methods}

\section{Nonadiabatic excited state molecular dynamics}

Nonadiabatic excited-state molecular dynamics (NEXMD) ${ }^{42,45}$ was used to model the photoexcited dynamics in the digon shown in Figure 1a. NEXMD is based on the mixed quantum classical fewest-switches surface hopping (FSSH) algorithm ${ }^{49}$ allowing modeling of electronic dynamics beyond the Born-Oppenheimer approximation as the system relaxes nonradiatively from a high-energy excited state to the lowest energy excited state. The nuclei evolve classically on a single adiabatic potential energy surface (PES) at any given time. The quantum mechanical description of electrons requires analytical excited state energies, gradients, and nonadiabatic couplings generated "on the fly". ${ }^{45,50-53}$ The electronic and nuclear systems are coupled by stochastic state switches in the PES for the nuclear trajectory based on the strength of the nonadiabatic derivative coupling. The collective electronic oscillator (CEO) $\operatorname{approach}^{54,55}$ is used to compute electronic excited states at the configuration interaction single (CIS) ${ }^{56}$ level of theory with a semiempirical AM1 ${ }^{57}$ Hamiltonian. This approach retains essential electronic correlations (e.g., excitonic effects) and has the benefit of being able to treat excited state dynamics in large conjugated systems. This method previously provided a realistic description of energy transfer in weakly coupled multichromophore systems. ${ }^{37-40,58,59}$ More details on the NEXMD implementation and parameters are reported elsewhere. ${ }^{43,45,60-62}$

\section{Transition density analysis}

The adiabatic wave function can be followed through the changes in the spatial localization of the transition density for the current state. Within the CEO approach, transition density matrices are written in terms of an atomic orbital $(\mathrm{AO})$ basis ${ }^{63,64}$ as $\left(\rho^{0 \alpha}\right)_{n m}=$ $\left\langle\phi_{\alpha}(r ; \mathbf{R}(t))\left|c_{m}^{+} c_{n}\right| \phi_{0}(r ; \mathbf{R}(t))\right\rangle$, subject to the normalization condition $\sum_{n, m}\left(\rho^{0 \alpha}\right)_{n m}^{2}=1$ for the CIS approximation. ${ }^{54}$ Here, $\phi_{0}(r ; \mathbf{R}(t))$ and $\phi_{\alpha}(r ; \mathbf{R}(t))$ are the ground and excited state 
adiabatic wave functions, respectively, AO basis functions are indexed by $n$ and $m$, and $c_{m}^{+}$and $c_{n}$ are creation and annihilation operators. Diagonal elements of $\rho^{0 \alpha}$ represent the net change in the electronic density on an atomic orbital when a ground to excited state transition occurs. The fraction of electronic transition density, denoted by $\delta$, localized on any segment $X$ is computed as the sum of atomic orbital contributions for each atom in the segment as follows $\delta_{X}^{\alpha}=\left(\rho^{0 \alpha}\right)_{X}^{2}=\sum_{n_{A} m_{A}}\left(\rho^{0 \alpha}\right)_{n_{A} m_{A}}^{2}$.

\section{Simulation details}

First, we perform a 1 ns equilibrated ground state $\left(S_{0}\right)$ molecular dynamics simulation using constant temperature Langevin dynamics at $300 \mathrm{~K}$, from which 500 snapshots are extracted at 2 ps intervals. These snapshots provide initial geometries and momenta for the subsequent NEXMD simulations. The NEXMD trajectories are started from these initial configurations after an instantaneous vertical excitation to an initial excited state $\alpha$ with frequency $\Omega_{\alpha}$ selected according to the Franck-Condon window defined as $g_{\alpha}(r, R)=f_{\alpha} \exp \left[-T^{2}\left(E_{l a s e r}-\right.\right.$ $\left.\Omega_{\alpha}\right)$ ], where $f_{\alpha}$ represents the normalized oscillator strength for the $\alpha$ state, and $E_{\text {laser }}\left(\mathrm{fs}^{-1}\right)$, is the energy of the simulated laser pulse centered at $423 \mathrm{~nm}$. We considered a Gaussian laser pulse, $f(t)=\exp \left(-t^{2} / 2 T^{2}\right)$, with $T=42.5$ fs corresponding to a FWHM (Full Width at Half Maximum) of 100 fs. Following the instantaneous vertical excitation, each of the 500 NEXMD trajectories was run for 500 fs using a Langevin thermostat ${ }^{65}$ to keep the temperature at $300 \mathrm{~K}$ with a friction coefficient of $20.0 \mathrm{ps}^{-1}$. Temperature effects mainly allow sampling of the initial ground-state conformational diversity of the molecule while coupling to the solvent (through the Langevin term) is not expected to affect the ultrafast intramolecular exciton redistribution after photoexcitation. ${ }^{66}$ Dielectric solvation effects of the experimental matrix were taken to be negligible, consistent with previous simulations and supported by spectral comparisons. ${ }^{67}$ Ten electronic states were included in the simulations. A classical time step of 0.1 fs was used for propagation of nuclei and a quantum time step of 0.025 fs to propagate the electronic degrees of freedom. In order to deal with trivial 
unavoided crossings, ${ }^{62}$ the quantum time step was further reduced by a factor of 40 in the vicinity of such crossings. Crucially, we do not observe any excessive dispersion interactions in either the ground or excited state dynamics which would lead the digon to collapse onto itself. ${ }^{67}$ The oligomers exhibited sufficient flexibility to give rise to a broad range of bending and torsional deformations.

\section{Acknowledgement}

We thank Philipp Wilhelm for providing the absorption spectrum of the material in Fig. 1. This work was performed in part at the Center for Nonlinear Studies (CNLS) and the Center for Integrated Nanotechnology (CINT), a U.S. Department of Energy and Office of Basic Energy Sciences user facility. We acknowledge support from the Los Alamos National Laboratory (LANL) Directed Research and Development funds (LDRD). This research used

resources provided by the Los Alamos National Laboratory Institutional Computing Program and CONICET, UNQ, ANPCyT (PICT- PICT-2014-2662). This work was supported by the US Department of Energy through the Los Alamos National Laboratory. Los Alamos National Laboratory is operated by Triad National Security, LLC, for the National Nuclear Security Administration of U.S. Department of Energy (Contract No. 89233218NCA000001).

\section{Supporting Information Available}

Comparison of experimental and simulated absorption spectra, Distributions of the initial transition density localization for states $S_{1}-S_{5}$, analysis of random localization within the chain that initially absorbs the most, number of changes in localization between the vertices. This material is available free of charge via the Internet at http://pubs.acs.org/. 


\section{References}

(1) Sun, K.; Xiao, Z.; Lu, S.; Zajaczkowski, W.; Pisula, W.; Hanssen, E.; White, J. M.; Williamson, R. M.; Subbiah, J.; Ouyang, J. et al. A Molecular Nematic Liquid Crystalline Material for High-Performance Organic Photovoltaics. Nature Comm. 2015, 6, 6013.

(2) Di, D.; Romanov, A. S.; Yang, L.; Richter, J. M.; Rivett, J. P. H.; Jones, S.; Thomas, T. H.; Abdi Jalebi, M.; Friend, R. H.; Linnolahti, M. et al. High-Performance Light-Emitting Diodes Based on Carbene-Metal-Amides. Science 2017, 356, 159-163.

(3) Qin, T.; Troisi, A. Relation between Structure and Electronic Properties of Amorphous MEH-PPV Polymers. J. Am. Chem. Soc. 2013, 135, 11247-11256.

(4) Dykstra, T. E.; Hennebicq, E.; Beljonne, D.; Gierschner, J.; Claudio, G.; Bittner, E. R.; Knoester, J.; Scholes, G. D. Conformational Disorder and Ultrafast Exciton Relaxation in PPV-family Conjugated Polymers. J. Phys. Chem. B 2009, 113, 656-667.

(5) Westenhoff, S.; Beenken, W. J. D.; Yartsev, A.; Greenham, N. C. Conformational disorder of conjugated polymers. J. Chem. Phys. 2006, 125, 154903.

(6) Albert, I. D. L.; Marks, T. J.; Ratner, M. A. Conformationally-induced geometric electron localization. Interrupted conjugation ; very large hyperpolarizabilities ; and sizable infrared absorption in simple twisted molecular chromophores. J. Am. Chem. Soc. 1997, 119, 3155-3156.

(7) Hagler, T. W.; Pakbaz, K.; Voss, K. F.; Heeger, A. J. Enhanced Order And Electronic Delocalization In Conjugated Polymers Oriented By Gel Processing In Polyethylene. Phys. Rev. B 1991, 44, 8652-8666.

(8) Lim, S. H.; Bjorklund, T. G.; Spano, F. C.; Bardeen, C. J. Exciton Delocalization and 
Superradiance in Tetracene Thin Films and Nanoaggregates. Phys. Rev. Lett. 2004, 92, $107402-4$.

(9) Bartholomew, G. P.; Bazan, G. C. Bichromophoric paracyclophanes: Models for interchromophore delocalization. Acc. Chem. Res. 2001, 34, 30-39.

(10) Van Averbeke, B.; Beljonne, D. Conformational Effects on Excitation Transport along Conjugated Polymer Chains. J. Phys. Chem. A 2009, 113, 2677-2682.

(11) Shi, T.; Li, H.; Tretiak, S.; Chernyak, V. Y. How Geometric Distortions Scatter Electronic Excitations in Conjugated Macromolecules. J. Phys. Chem. Lett. 2014, 5, 39463952.

(12) Nayyar, I. H.; Batista, E. R.; Tretiak, S.; Saxena, A.; Smith, D. L.; Martin, R. L. Role of Geometric Distortion and Polarization in Localizing Electronic Excitations in Conjugated Polymers. J. Chem. Theory Comput. 2013, 9, 1144-1154.

(13) Magyar, R.; Tretiak, S.; Gao, Y.; Wang, H.-L.; Shreve, A. A Joint Theoretical and Experimental Study of PhenyleneAcetylene Molecular Wires. Chem. Phys. Lett. 2005, 401, 149-156.

(14) Nguyen, T.-Q.; Martini, I. B.; Liu, J.; Schwartz, B. J. Controlling Interchain Interactions in Conjugated Polymers: The Effects of Chain Morphology on Exciton-Exciton Annihilation and Aggregation in MEH-PPV Films. J. Phys. Chem. B 2000, 104, 237255.

(15) Becker, K.; Da Como, E.; Feldmann, J.; Scheliga, F.; Thorn Csányi, E.; Tretiak, S.; Lupton, J. M. How Chromophore Shape Determines the Spectroscopy of PhenyleneVinylenes: Origin of Spectral Broadening in the Absence of Aggregation. J. Phys. Chem. B 2008, 112, 4859-4864. 
(16) Campbell, I. H.; Rubin, S.; Zawodzinski, T. A.; Kress, J. D.; Martin, R. L.; Smith, D. L.; Barashkov, N. N.; Ferraris, J. P. Controlling Schottky energy barriers in organic electronic devices using self-assembled monolayers. Phys. Rev. B 1996, 54, R14321.

(17) Waters, M. J.; Hashemi, H.; Shi, G.; Kioupakis, E.; Kieffer, J. Predictive Simulations for Tuning Electronic and Optical Properties of SubPc Derivatives. arXiv 2017, 1705.00409 .

(18) Jäger, L.; Schmidt, T. D.; Brütting, W. Manipulation and control of the interfacial polarization in organic light-emitting diodes by dipolar doping. AIP Adv. 2016, 6 , 095220 .

(19) Noguchi, Y.; Miyazaki, Y.; Tanaka, Y.; Sato, N.; Nakayama, Y.; Schmidt, T. D.; Brütting, W.; Ishii, H. Charge accumulation at organic semiconductor interfaces due to a permanent dipole moment and its orientational order in bilayer devices. J. Appl. Phys. 2012, 111, 114508.

(20) Sampat, S.; Mohite, A. D.; Crone, B.; Tretiak, S.; Malko, A. V.; Taylor, A. J.; Yarotski, D. A. Tunable Charge Transfer Dynamics at Tetracene/LiF/C60 Interfaces. J. Phys. Chem. C 2015, 119, 1286-1290.

(21) Brand, C.; Meerts, W. L.; Schmitt, M. How and Why Do Transition Dipole Moment Orientations Depend on Conformer Structure? J. Phys. Chem. A 2011, 115, 96129619.

(22) Obaid, R.; Leibscher, M. A molecular symmetry analysis of the electronic states and transition dipole moments for molecules with two torsional degrees of freedom. J. Chem. Phys. 2015, 142, 064315.

(23) Senes, A.; Meskers, S. C. J.; Dijkstra, W. M.; van Franeker, J. J.; Altazin, S.; Wilson, J. S.; Janssen, R. A. J. Transition dipole moment orientation in films of solution 
processed fluorescent oligomers: investigating the influence of molecular anisotropy. $J$. Mater. Chem. C 2016, 4, 6302-6308.

(24) Liehm, P.; Murawski, C.; Furno, M.; Lüssem, B.; Leo, K.; Gather, M. C. Comparing the emissive dipole orientation of two similar phosphorescent green emitter molecules in highly efficient organic light-emitting diodes. Appl. Phys. Lett. 2012, 101, 253304.

(25) Hestand, N. J.; Spano, F. C. Moleculate Aggregate Photophysics beyond the Kasha Model: Novel Desing Principles for Organic Materials. Acc. Chem. Res. 2017, 50, $341-350$.

(26) Hestand, N. J.; Spano, F. C. The Effect of Chain Bending on the Photophysical Properties of Conjugated Polymers. J. Phys. Chem. B 2014, 118, 8352-8363.

(27) Bradforth, S. E.; Jinenez, R.; van Mourik, F.; van Grondelle, R.; Fleming, G. R. Excitation Transfer In The Core Light-Harvesting Complex (Lh-1) Of RhodobacterSphaeroides : An Ultrafast Fluorescence Depolarization And Annihilation Study. J. Phys. Chem. 1995, 99, 16179-16191.

(28) Camacho, R.; Tubasum, S.; Southall, J.; Cogdell, R. J.; Sforazzini, G.; Anderson, H. L.; Pullerits, T.; Scheblykin, I. G. Fluorescence Polarization Measures Energy Funneling in Single Light-Harvesting Antennas - LH2 vs Conjugated Polymers. Sci. Rep. 2015, $5,15080$.

(29) Yong, C.-K.; Parkinson, P.; Kondratuk, D.; Chen, W.-H.; Stannard, A.; Summerfield, A.; Sprafke, J.; O’Sullivan, M.; Beton, P.; Anderson, H. et al. Ultrafast Delocalization of Excitation in Synthetic Light-Harvesting Nanorings. Chem. Sci. 2015, 6, $181-189$.

(30) Johnson, J. M.; Chen, R.; Chen, X.; Moskun, A. C.; Zhang, X.; Hogen-Esch, T. E.; Bradforth, S. E. Investigation of Macrocyclic Polymers as Artificial Light Harvesters: 
Subpicosecond Energy Transfer in Poly(9,9-Dimethyl-2-Vinylfluorene). J. Phys. Chem. B 2008, 112, 6367-16381.

(31) Aggarwal, A. V.; Thiessen, A.; Idelson, A.; Kalle, D.; Wursch, D.; Stangl, T.; Steiner, F.; Jester, S.-S.; Vogelsang, J.; Hoger, S. et al. Fluctuating Exciton Localization in Giant

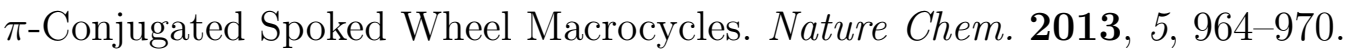

(32) Varnavski, O.; Samuel, I. D. W.; Palsson, L.-O.; Beavington, R.; Burn, P. L.; Goodson, T. Investigations of Excitation Energy Transfer and Intramolecular Interactions in a Nitrogen Corded Distrylbenzene Dendrimer System. J. Chem. Phys. 2002, 116, 8893.

(33) Collini, E.; Scholes, G. Coherent intrachain energy migration in a conjugated polymer at room temperature. Science 2009, 323, 369-373.

(34) Schmid, S. A.; Yim, K. H.; Chang, M. H.; Zheng, Z.; Huck, W. T. S.; Friend, R. H.; Kim, J. S.; Herz, L. M. Polarization anisotropy dynamics for thin films of a conjugated polymer aligned by nanoimprinting. Phys. Rev. B 2008, 77, 115338.

(35) Yamazaki, I.; Akimoto, S.; Yamazaki, T.; Sato, S.-I.; Sakata, Y. Oscillatory Exciton Transfer in Dithiaanthracenophane: Quantum Beat a Coherent Photochemical Process in Solution. J. Phys. Chem. A 2002, 106, 2122-2128.

(36) Zhu, F.; Galli, C.; Hochstrasser, R. M. The Real-Time Intramolecular Electronic Excitation Transfer Dynamics of 9',9-Bifluorene and 2',2-Binaphtyl in Solution. J. Chem. Phys. 1993, 98, 1042-1057.

(37) Galindo, J. F.; Atas, E.; Altan, A.; Kuroda, D. G.; Fernandez-Alberti, S.; Tretiak, S.; Roitberg, A. E.; Kleiman, V. D. Dynamics of Energy Transfer in a Conjugated Dendrimer Driven by Ultrafast Localization of Excitations. J. Am. Chem. Soc. 2015, 137, 11637-11644. 
(38) Ondarse-Alvarez, D.; Komurlu, S.; Roitberg, A. E.; Pierdominici-Sottile, G.; Tretiak, S.; Fernandez-Alberti, S.; Kleiman, V. D. Ultrafast Electronic Energy Relaxation in a Conjugated Dendrimer Leading to Inter-Branch Energy Redistribution. Phys. Chem. Chem. Phys. 2016, 18, 25080-25089.

(39) Alfonso Hernandez, L.; Nelson, T.; Tretiak, S.; Fernandez-Alberti, S. Photoexcited Energy Transfer in a Weakly Coupled Dimer. J. Phys. Chem. B 2015, 119, 7242-7252.

(40) Alfonso Hernandez, L.; Nelson, T.; Gelin, M. F.; Lupton, J. M.; Tretiak, S.; Fernandez-Alberti, S. Interference of Interchromophoric Energy-Transfer Pathways in pi-Conjugated Macrocycles. J. Phys. Chem. Lett. 2016, 7, 4936-4944.

(41) Wilhelm, P.; Vogelsang, J.; Poluektov, G.; Schönfelder, N.; Keller, T. J.; Jester, S.-S.; Höger, S.; Lupton, J. M. Molecular Polygons Probe the Role of Intramolecular Strain in the Photophysics of pi-Conjugated Chromophores. Angew. Chem. Int. Ed. Engl. 2017, $56,1234-1238$.

(42) Sifain, A. E.; Bjorgaard, J. A.; Nelson, T. R.; Nebgen, B. T.; White, A. J.; Gifford, B. J.; Gao, D. W.; Prezhdo, O. V.; Fernandez-Alberti, S.; Roitberg, A. E. et al. Photoexcited Nonadiabatic Dynamics of Solvated PushPull $\pi$-Conjugated Oligomers with the NEXMD Software. J. Chem. Theory Comput. 2018, 14, 3955-3966.

(43) Nelson, T.; Fernandez-Alberti, S.; Roitberg, A. E.; Tretaik, S. Nonadiabatic ExcitedState Molecular Dynamics: Modeling Photophysics in Organic Conjugated Materials. Acc. Chem. Res. 2014, 47, 1155-1164.

(44) Nelson, T.; Fernandez-Alberti, S.; Roitberg, A. E.; Tretiak, S. Electronic Delocalization, Vibrational Dynamics, and Energy Transfer in Organic Chromophores. J. Phys. Chem. Lett. 2017, 8, 3020-3031.

(45) Nelson, T.; Fernandez-Alberti, S.; Chernyak, V.; Roitberg, A. E.; Tretiak, S. Nona- 
diabatic Excited-State Molecular Dynamics Modeling of Photoinduced Dynamics in Conjugated Molecules. J. Phys. Chem. B 2011, 115, 5402-5414.

(46) Tretiak, S.; Mukamel, S. Density Matrix Analysis and Simulation of Electronic Excitations in Conjugated and Aggregated Molecules. Chem. Rev. 2002, 102, 3171-3212.

(47) Nelson, T. R.; Ondarse-Alvarez, D.; Oldani, N.; Rodriguez-Hernandez, B.; AlfonsoHernandez, L.; Galindo, J. F.; Kleiman, V. D.; Fernandez-Alberti, S.; Roitberg, A. E.; Tretiak, S. Coherent Exciton-Vibrational Dynamics and Energy Transfer in Conjugated Organics. Nature Comm. 2018, 9, 2316.

(48) Demchenko, A. P.; Tomin, V. I.; Chou, P.-T. Breaking the Kasha Rule for More Efficient Photochemistry. Chem. Rev. 2017, 117, 13353-13381.

(49) Tully, J. Molecular Dynamics with Electronic Transitions. J. Chem. Phys. 1990, 93, 1061-1071.

(50) Tavernelli, I.; Curchod, B. F. E.; Laktionov, A.; Rothlisberger, U. Nonadiabatic Coupling Vectors for Excited States within Time-Dependent Density Functional Theory in the Tamm-Dancoff Approximation and Beyond. J. Chem. Phys. 2010, 133, 194104.

(51) Furche, F. On the Density Matrix Based Approach to Time-Dependent Density Functional Response Theory. J. Chem. Phys. 2001, 114, 5982-5992.

(52) Furche, F.; Ahlrichs, R. Adiabatic Time-Dependent Density Functional Methods for Excited State Properties. J. Chem. Phys. 2002, 117, 7433-7447.

(53) Tretiak, S.; Chernyak, V.; Mukamel, S. Recursive Density-Matrix-Spectral-Moment Algorithm for Molecular Nonlinear Polarizabilities. J. Chem. Phys. 1996, 105, 89148928.

(54) Tretiak, S.; Isborn, C.; Niklasson, A.; Challacombe, M. Representation Independent 
Algorithms for Molecular Response Calculations in Time-Dependent Self-Consistent Field Theories. J. Chem. Phys. 2009, 130, 054111.

(55) Chernyak, V.; Schulz, M. F.; Mukamel, S.; Tretiak, S.; Tsiper, E. V. Krylov-space algorithms for time-dependent Hartree-Fock and density functional computations. $J$. Chem. Phys. 2000, 113, 36-43.

(56) Thouless, D. J. The Quantum Mechanics Of Many-Body Systems; Academic Press: New York, 1972.

(57) Dewar, M. J. S.; Zoebisch, E. G.; Healy, E. F.; Stewart, J. J. P. AM1: A New General Purpose Quantum Mechanical Molecular Model. J. Am. Chem. Soc. 1985, 107, 39023909.

(58) Ortiz, W.; Krueger, B. P.; Kleiman, V. D.; Krause, J. L.; Roitberg, A. E. Energy Transfer in the Nanostar: The Role of Coulombic Coupling and Dynamics. J. Phys. Chem. B 2005, 109, $11512-11519$.

(59) Ondarse-Alvarez, D.; Oldani, N.; Tretiak, S.; Fernandez-Alberti, S. Computational Study of Photoexcited Dynamics in Bichromophoric Cross-Shaped Oligofluorene. J. Phys. Chem. A 2014, 118, 10742-10753.

(60) Nelson, T.; Fernandez-Alberti, S.; Chernyak, V.; Roitberg, A.; Tretiak, S. Nonadiabatic Excited-State Molecular Dynamics: Numerical Tests of Convergence and Parameters. J. Chem. Phys. 2012, 136, 054108.

(61) Nelson, T.; Fernandez-Alberti, S.; Roitberg, A. E.; Tretaik, S. Nonadiabatic ExcitedState Molecular Dynamics: Treatment of Electronic Decoherence. J. Chem. Phys. 2013, $138,224111$.

(62) Fernandez-Alberti, S.; Roitberg, A.; Nelson, T.; Tretiak, S. Identification of Unavoided 
Crossings in Nonadiabatic Photoexcited Dynamics Involving Multiple Electronic States in Polyatomic Conjugated Molecules. J. Chem. Phys. 2012, 137, 014512.

(63) Tretiak, S.; Chernyak, V.; Mukamel, S. Two-Dimensional Real-Space Analysis of Optical Excitations in Acceptor-Substituted Carotenoids. J. Am. Chem. Soc. 1997, 119, $11408-11419$.

(64) Tretiak, S.; Chernyak, V.; Mukamel, S. Collective Electronic Oscillators for Nonlinear Optical Response of Conjugated Molecules. Chem. Phys. Lett. 1996, 259, 55-61.

(65) Paterlini, M.; Ferguson, D. Constant Temperature Simulations using the Langevin Equation with Velocity Verlet Integration. Chem. Phys. 1998, 236, 243-252.

(66) Shenai, P. M.; Fernandez-Alberti, S.; Bricker, W. P.; Tretiak, S.; Zhao, Y. Internal Conversion and Vibrational Energy Redistribution in Chlorophyll A. J. Phys. Chem. $B$ 2016, 120, 49-58.

(67) Allolio, C.; Stangl, T.; Eder, T.; Schmitz, D.; Vogelsang, J.; Höger, S.; Horinek, D.; Lupton, J. M. H-Aggregation Effects between pi-Conjugated Chromophores in Cofacial Dimers and Trimers: Comparison of Theory and Single-Molecule Experiment. J. Phys. Chem. B 2018, 122, 6431-6441. 